\title{
Un vitalismo deleuziano: bosquejos sobre el diagrama
}

\author{
A Deleuzian vitalism: sketches about diagram
}

\section{Patricio Landaeta ${ }^{[a]}$, Braulio Rojas $^{[a]}$, Ana María Cristi ${ }^{[b]^{*}}$}

[a] Universidad de Playa Ancha, Valparaíso, Chile

[b] Pontificia Universidad Católica de Valparaíso, Valparaíso, Chile

\section{Resumen}

El problema del vitalismo en la filosofía de Gilles Deleuze recorre toda su obra y se presenta fundamentalmente como un llamado a liberar la vida de toda forma de sujeción. Así, el vitalismo no debe ser tomado por un contenido entre otros, sino por, digámoslo así, la expresión de su propia perspectiva, que toma la filosofía por una creación de conceptos. Por ello, la presentación de lo que implica un vitalismo deleuziano exige que se plantee en el marco de su pensamiento para, evitando el mero comentario, repetir su filosofía. Siguiendo tal idea, en un primer momento, nos adentramos en la filosofía de Nietzsche, específicamente en la distinción que se presenta entre una historia monumental, una historia anticuaria y una historia crítica, que discurren acerca de los preceptos de una historia útil para la vida, hasta captar, en un segundo momento, como se ven materializados en el propio trabajo de Deleuze estas apreciaciones hechas por Nietzsche

PL: Doctor en Filosofía, e-mail: patricio.landaeta@upla.cl

BL: Doctor en Filosofía, e-mail: braulio.rojas@upla.cl

AMC: Licenciada en Filosofía, e-mail: ana.cristi.c@gmail.com 
en Sobre la utilidad y el perjuicio de la historia para la vida [II Intempestiva]: Deleuze equilibra en su trabajo la agudeza del metafísico, la erudición del historiador y la potencia destructiva del crítico, con vistas a procurar un saber y una experiencia al servicio de lo que potencia la vida, saber y experiencia que permiten pensar el tránsito que va desde lo que estamos dejando de ser, nuestro pasado inmediato, a lo que estamos deviniendo, nuestro futuro inmediato. Finalmente, tras esta preparación, se expone a partir de la noción de diagrama las principales consecuencias del vitalismo de Deleuze, expresión de una filosofía al servicio de la vida o, propiamente, diagnóstico de nuevas posibilidades de vida en lo que se escapa al presente.

Palabras clave: Deleuze. Nietzsche. Foucault. Vitalismo. Diagrama.

\section{Abstract}

The problem of vitalism in philosophy of Gilles Deleuze wanders his entire work implicating an appeal: to liberate life from any kind of subjugation. Vitalism is not mere content, since vitalism expresses in Deleuze his own point of view about philosophy as creation of concepts. Thus, a presentation of what implicates a Deleuzian vitalism demands to be raised in the context of his thought, avoiding interpretation, to, finally, repeat his philosophy. Following this idea, we move after into the reading of Nietzsche's philosophy, specifically into the conceived difference among monumental history, antiquarian history and critical history, argued to delimitate basics precepts for a useful history for life. Then, in a second moment, ideas and comments created at II Untimely By Nietzsche will be developed as part of Deleuze own work. Deleuze try to balance in his work metaphysics, history of philosophy and critic, in order to produce a philosophy useful for life, considering the difference between what we are ceasing to be, our immediate past, and what we are becoming, our immediate future. Finally, after this preparation, it is exposed, through the notion of diagram, the major consequences of vitalism in Gilles Deleuze, expression of philosophy useful for life or diagnosis of new possibilities for life in what scape of present.

Keywords: Deleuze. Nietzsche. Foucault. Vitalism. Diagram. 


\section{Introducción: cómo componer un vitalismo deleuziano}

Toda la filosofía de Deleuze es un himno a la vida, una afirmación de la vida. Y nadie supo mejor que él vivir filosóficamente hasta el extremo límite (SHERER, 2012, 16).

Un vitalismo anima la filosofía de Gilles Deleuze (PROTEVI, 2012; SCHÉRER, 2012) que se canaliza en la reconocida exhortación a "liberar" la vida de aquello que la aprisiona disminuyendo su potencia (1996a, p. 228). Foucault (2000), en el célebre prefacio de la traducción al inglés de El Anti-Edipo. Capitalismo y esquizofrenia, enfatiza que el texto que presenta constituye un libro de ética sin parangón, un verdadero "arte de vivir" que promueve una "introducción para la vida no fascista" (p. XII). Y no es anecdótico que, como un ajuste de cuentas, ya casi agotando sus fuerzas, un último texto breve, L'immanence: une vie... (1995), revisite con una especie de "misticismo ateo" los principales conceptos que componen y articulan su idea de vitalismo (SCHÉRER, 2012, p. 30-31), modelando para sus lectores un precioso legado ${ }^{1}$. En efecto, "vitalismo" y "vida" resultan elementos tan esenciales, que, a partir de su vínculo, la excéntrica historia de la filosofía propuesta por el francés (esa que reúne en un mismo itinerario figuras dispares como Lucrecio, Hume, Spinoza, Leibniz, Nietzsche, Bergson y Foucault) se definiría por declarar que pensar, en sus múltiples facetas, constituye una actividad de resistencia ejercida contra toda forma de sometimiento, llegando a constituirse como una especie de nueva etología. En Nietzsche y la filosofía de 1962 un joven Deleuze sostiene: "pensar significa esto: inventar, crear nuevas posibilidades de vida” (1983, p. 115). Y casi treinta años más tarde, en 1988, confiesa: "todo cuanto he escrito - al menos así lo espero - ha sido vitalista" (1996a, p. 228). No obstante, ¿cómo nos interpela y qué puede expresar el vitalismo deleuziano cuando distintas concepciones de la vida

1 René Schérer (2012) aborda la diferencia existente entre el vitalismo deleuziano, impersonal y asubjetivo, y las "filosofías de la vida", demasiado humanistas, al permanecer ancladas en la conciencia y el sujeto: "Deleuze piensa la vida pre- $y$ a-subjetiva, pre- e in-orgánica, pre-y no-individual. La extiende a las cosas, a las formas, a los abstractos: la vida de 'la línea abstracta"' (p. 23). 
convergen en el centro de la querella biopolítica? ${ }^{2}$ Con una interrogante que nos fuerza a pensar nuestra situación nos adentramos y, al mismo tiempo, tomamos distancia del pensamiento de Deleuze. Puesto que si queremos reparar en la actualidad del vitalismo, tendríamos que evitar tomar por objeto su filosofía y "calcar" lo que cursos, entrevistas y libros expresan al respecto, como si el vitalismo se tratase de un mero contenido. Al contrario, si queremos ahondar en su actualidad, hemos de aproximarnos a su esclarecimiento desde nuestra situación, a fin de confrontar paralelamente la filosofía de Deleuze y la crisis de nuestro tiempo, cuya amenaza principal sea tal vez el sojuzgamiento de la vida a manos de un poder que deja de ejercer su influjo en espacios cerrados, como la escuela, el hospital y la cárcel, propios de las sociedades disciplinarias. En ese sentido: "las sociedades disciplinarias son nuestro pasado inmediato, lo que estamos dejando de ser" (DELEZUE, 1996a, p. 277). En lugar de ellas, se advierte por doquier la imposición de una nueva estrategia, que se avista como nuestro futuro inmediato, las "sociedades de control" (DELEZUE, 1996a, p. 278).

Se indagará en el vitalismo deleuziano desplazando a Deleuze para ofrecer en un acercamiento a distancia un nuevo montaje de sus ideas que permita repetir su pensamiento. Al proceder de esta manera no hacemos más que asegurarnos de seguir los propios planteamientos deleuzianos. Deleuze quiere comprender, por ejemplo, a Foucault, pero comprender no quiere comentar su obra (GROS, 1995, p. 53). Porque, el ejercicio filosófico, en el modo en que queda ya expresado en Diferencia y repetición (1968), no es ni estrictamente interpretativo,

2 La biopolítica ha sido abordada por Michel Foucault brevemente en la parte final de La voluntad de saber e in extenso en su curso del Collège de France (1978-1979): El nacimiento de la biopolítica. Por Biopolítica ha de entenderse: "la manera como se ha procurado, desde el siglo XVIII, racionalizar los problemas planteados a la práctica gubernamental por los fenómenos propios de un conjunto de seres vivos constituidos como población: salud, higiene, natalidad, longevidad, razas" (FOUCAULT, 2007, p. 359). Sin embargo, el problema no se agota en el planteamiento de Foucault, contando contemporáneamente con los aportes de Giorgio Agamben, en su examen de la nuda vida en Homo sacer (1998); Antonio Negri y Michel Hardt con su trabajo sobre las formas de control en el mundo globalizado en Imperio (2005); Roberto Esposito y su lectura de la biopolítica afirmativa desde una deriva deleuziana a partir de Bíos. Biopolítica y filosofía (2006). Soto García, destacando la impronta deleuziana del pensamiento de Esposito, distingue entre una dimensión afirmativa "que preserva y mantiene la vida y una negativa, que "desde su carácter inmunitario [... tiene como primer propósito la protección de la vida" (SOTO GARCíA, 2015, p. 81). 
ni exegético. Al contrario, debe ser creativo y hasta intempestivo (DELEUZE, 2002). Por ello, ha de entenderse que hacer historia de la filosofía, abordando el trabajo de un filósofo cualquiera, es intentar hacer funcionar sus conceptos, trastocando muchas veces los límites de la fidelidad con el autor. En palabras de Deleuze: "sería necesario que la exposición, en historia de la filosofía, actúe como verdadero doble y contenga la modificación máxima propia del doble" (2002, p. 18-19). El asunto, entonces, no concluye en el uso de los conceptos en los términos concebidos por una historia "interior" del pensamiento, sino en establecer cómo los conceptos ajenos pueden también jugarse en nuevas coordenadas históricas sin que, claro está, se reduzcan a ella (DELEUZE, 1996a, p. 172). De otro modo, la cuestión es saber cómo los conceptos creados en otra contingencia podrían contribuir tentativamente, mediante una torsión, al esclarecimiento de un problema que nos interpela en la actualidad. Porque buscar la exactitud, siguiendo el afán de reproducir con precisión ideas y conceptos creados por filósofos de antaño o por nuestros contemporáneos, nos conduce a dos inconvenientes: por un lado, confundir el ejercicio del pensamiento con una contribución estéril a la historia de la filosofía, cerrada y vuelta sobre sí, que se autoafirma en el academicismo; y, por otro, creer que la filosofía se hace sobre la filosofía y no sobre algo otro, como es el afuera o la Vida.

Un filosofar creativo, más cercano a la ficción literaria que a la filosofía universitaria, que intenta constantemente poner en juego un montaje que amenaza con subvertir la identidad de los conceptos, no implica ninguna renuncia, tampoco una traición a la filosofía. Al contrario, un ejercicio de este tipo no tiene nada de irreverente, ni tampoco su violencia debe entenderse como si encarnase un gesto irónico, condenado a desaparecer tras conseguir su efecto: el escándalo. El "travestismo" de Deleuze implica una afirmación que debe ser comprendida en el marco que su propio vitalismo instala. Así lo señala François Zourabichvili:

Los autores trabajados por Deleuze tienen menos, para él, función de padres o maestros que de "intercesores" [...] liberando un discurso 
indirecto libre: es este discurso original [...], donde cada cual deviene otro [...]. En tales condiciones [...] todo título de propiedad viene a ser tachado (brouillé), o resulta de una perturbación (brouillage), y el propio mimetismo viene a resultar una impostura (2013, p. 377).

Esta afirmación de un cercano a Deleuze nos conduce a la siguiente pregunta, volviendo con el problema que nos convoca, el problema de un vitalismo deleuziano: ¿por qué ese gesto de construir una historia de la filosofía desde lo tachado (brouillé) y la perturbación (brouillage) definiría una actitud que entrecruza vida y pensamiento? Intentaremos responder las inquietudes planteadas, desplazando la filosofía de Gilles Deleuze como decíamos en un comienzo. En un primer momento, a partir de Nietzsche, sumergiéndonos en su Sobre la utilidad y perjuicio de la historia para la vida. II Intempestiva, para, en un segundo momento, leer la filosofía del francés como un contrapunto o equilibrio de las tres formas de hacer historia que distingue el texto. Finalmente, este itinerario debiese permitirnos producir un nuevo retrato de la filosofía de Deleuze (declarada en guerra contra las nuevas formas de sujeción que se avistan) para establecer en el concepto de diagrama ${ }^{3}$ el aspecto clave del vitalismo

3 La idea de "diagrama" y sobre todo la función "diagramática" y el "diagramatismo", atribuido a ciertos procesos de orden "molecular", pueden ser localizados en distintos pasajes de la producción escrita de Deleuze y Guattari. Si desprendemos su importancia de su constante reiteración, se diría que son términos claves en el aparato conceptual de ambos filósofos. Sin embargo, la delimitación 0 extensión de tales ideas y conceptos no pueden tenerse como algo consabido. La noción de diagrama emerge en sus colaboraciones por primera vez en Mil mesetas. Capitalismo y esquizofrenia 2, en el año 1981. № obstante, cabe marcar primeramente su emergencia en la producción del propio Deleuze en solitario. En un artículo de 1975 dedicado a Foucault: No un escritor, un nuevo cartógrafo, hace su aparición la idea de diagrama, y, más tarde, en el curso "Pintura", impartido en la Universidad Paris VIII, base del texto Francis Bacon. Lógica de la sensación, publicado el año 1981, el concepto adquiere notoria visibilidad y experimenta un desplazamiento respecto de su primera formulación de corte foucaultiana. Más tarde, vemos que el asunto de los "diagrama de poder" es investido de una fuerte importancia en el curso dictado por Deleuze sobre Foucault en la misma universidad, curso que se complementa con la publicación del texto Foucault en 1986, donde el concepto vuelve a aparecer marcando acercamientos y distancias con el pensamiento de Foucault (sobre todo en relación con el problemas de las fuerzas). Paralelamente, esta noción adquiere la forma de un insistente concepto, un especie de sello que distingue la "ciencia nómade", que ocupa un lugar destacable en la actividad intelectual de Guattari, como se advierte en Cartografías esquizoanalíticas de 1989, Caosmosis de 1992, y Micropolítica. Cartografías del deseo, en colaboración con Suely Rolnik y publicado varios años después de la muerte de Guattari en 2005. En cada una de estas obras es posible advertir de manera general el valor atribuido a los calificativos "diagramático" y "diagramatismo", como opuestos a todo lo estable, "sedentario"y esquemático de las ciencias "mayores". Sobre el origen del término, tenemos que destacar una doble filiación. En primer lugar, el término sería tomado de la obra de Foucault, como deja ver Deleuze en el citado artículo de 1975. En Vigilary

Rev. Filos., Aurora, Curitiba, v. 29, n. 46, p. 73-94, jan./abr. 2017 
deleuziano y uno de las principales herramientas para confrontar aquello que estamos deviniendo como sociedad.

\section{Nietzsche y la(s) historia(s)}

Nietzsche no es un padre para Deleuze, pero su influencia es profunda. Junto con Spinoza es sin duda el filósofo que le acompaña en su itinerario crítico y clínico. De entrada, el título del escrito de Nietzsche, Sobre la utilidad y perjuicio de la historia para la vida, insinúa una primera y primaria correspondencia con nuestra propuesta, que interroga acerca de la actualidad del vitalismo deleuziano o, si se quiere, incluso por la propia utilidad de la filosofía de Deleuze para la vida. Al ingresar en el texto la sintonía se advierte aún con mayor fuerza, dado que el autor del Anticristo lanza una clara exhortación: necesitamos la historia para la vida y para la acción, por tanto, necesitamos comprenderla alejados o contra su uso científico o docto, que poco o nada sabe de aquello que potencia o "vivifica". En sus palabras: "la Historia, pensada como ciencia pura y convertida en Soberana, sería una especie de conclusión de la vida, un ajuste final de cuentas. Solo si la educación histórica va acompañada de una poderosa y nueva corriente vital [...] es algo saludable y prometedora de futuro" $(2013$, p. 50).

Acorde con esta máxima, que arremete contra el saber puro de la historia, la II Intempestiva, yace marcada por el mito del hombre que quiere

castigar, publicado ese mismo año, se dice que el panóptico es un diagrama de poder, entendiendo que éste implica, en cuanto espacio arquitectónico polivalente, una intervención del poder en la vida cotidiana de una multiplicidad de individuos, sin pretensión de ejercer la mera coerción, sino dando lugar a una visibilidad generalizada, que permite la vigilancia, la disciplina, así como el cuidado y, sobre todo, que ese grupo humano se ofrezca, se abra al conocimiento (Foucault, 2002, p.178). Tal definición llevará, pues, a Deleuze a concluir, en un primer momento, la identificación de "diagrama" y "poder", cuestión que más tarde variará, a partir de Francis Bacon: lógica de la sensación, acentuándose ese cambio aún más en Foucault, al establecer el vínculo entre el diagrama y un "conjunto de fuerzas", como se verá más adelante en este artículo. En segundo lugar, Guattari aparta el término del horizonte político o "micropolítico" foucaultiano para destacar su procedencia lógica o lingüística (sin por ello dejar de investir el término de una fuerte carga política), atribuyéndolo a Charles Pierce. Para Guattari, siguiendo a Pierce, a diferencia de las "redundancias semiológicas", que representan 0 establecen un equivalente real sin asidero, las "interacciones diagramáticas" son sistemas de signos que trabajan directamente con las realidades que abordan y que, inclusive, producen su objeto (Guattari, 2006, 369). 
liberarse de su pasado. Brevemente, de acuerdo a este mito, el hombre se ve a sí mismo amarrado al pasado y envidia al animal y su confinamiento en el presente, porque viviendo de manera no-histórica, olvida sin esfuerzo cada instante que pasa. Por ello, cuando descubre dentro de sí mismo un ser para el cual el pasado no es nada, hace a un lado esa envidia primera para rendirse ante la imagen del niño, que no tiene ni pasado que recordar ni memoria que padecer. Finalmente, este mito ofrecerá al lector una paradójica lección: la falta de olvido impide ser feliz y, en consecuencia, impide también vivir, ya que sólo la felicidad "vivifica". Así, quien resultara incapaz de olvidar, afirmará Nietzsche, no llegará a ser feliz, ni logrará hacer felices a los demás. No obstante, y he ahí la terrible enseñanza de este mito, quien como el niño se vea arrastrado por el devenir no podrá llegar a creer en su propia existencia, ya que sin tener relación alguna con el pasado se perderá en ese flujo perpetuo que encarna el devenir. En concordancia con el mito, entonces, la historia útil para la vida, alejada del uso científico y docto, serpentea o deriva entre la falta de olvido del hombre y el olvido absoluto de la bestia, teniendo por ambigua efigie - tan lejos, tan cerca - la imagen del niño, el ser que aún no tiene tiempo que olvidar ni pasado que recordar (NIETZSCHE, 2013, p. 40-42).

¿A fuerza de qué renuncias y mediante qué gestos se alcanza esta historia útil para la vida? Consciente de los riesgos que entraña tamaña empresa, el filósofo - quien muchas veces dice emular a un médico de la cultura - anticipa como estrategia establecer un contrapunto entre los tres modos habituales de hacer historia y hacer uso del pasado, es decir, un equilibrio entre la "historia monumental" que extrae un modelo del pasado, entre la "historia anticuaria" que conserva el pasado y la "historia crítica" que, sirviéndose de un gesto demasiado violento, aunque con lúcidos objetivos, destruye el pasado en su lucha por/con el presente. Al revisar con más detalle, se advierte que la historia monumental, observando las grandes gestas del pasado, recoge un modelo a imitar para que quienes sean educados en su seno lleguen a convertirse en sujetos capaces de grandes proezas. Este modo de concebir la historia tendría gran importancia para Nietzsche, pues como paideia, como alimento o humus en el que crece una cultura, engendra hombres capaces de hazañas 
imposibles. No obstante, y he ahí el problema, ocurre que bajo su alero se cree que los hechos son efectos "en sí", creencia que, para Nietzsche, no resiste la más mínima dosis de genealogía (2013, p. 56-57) . La historia anticuaria, en el cuidado y veneración del pasado, atesora intacta la grandeza de lo antiguo y, perdiendo toda sensibilidad para apreciar el presente, desprecia lo nuevo (2013, p. 63). Este uso de la historia, en efecto, conserva el pasado, que sólo pervive momificado y disponible para los hombre en su intocable lejanía (2013, p. 63-64). Finalmente, la historia crítica, el más radical de los tres modos o usos de la historia, es puesta en juego para liberar a los hombres - y la propia vida - de las ataduras del pasado. Así lo escribe Nietzsche: "es menester que el hombre, para poder vivir, tenga la fuerza [de] destruir y liberarse del pasado" (2013, p. 65). Ahora bien, si esta violencia contra el pasado es necesaria y útil para la vida, también es cierto que somos, en tanto criaturas del presente, resultado, efecto o factura del pasado, fruto de sus aberraciones, pasiones y errores (2013, p. 66). Por tanto, la actitud que tengamos contra el pasado es una actitud que finalmente tenemos frente a nosotros mismos. Nietzsche es enfático al afirmar y distinguir la utilidad y exceso que conforma y nutre cada una de estas actitudes frente al pasado. Por ello, no dándoles la espalda, sino a partir de la búsqueda de un buen equilibrio entre estas tres perspectivas sería posible contrarrestar la amenaza que ejercen las piedras de un "saber puro", que indigesta a los hombres y agota su cultura (2013, p. 68). En efecto, frente al exceso que nutre cada uno de estos modos de hacer historia, se sostiene que una historia que tiene por fin servir a la vida es aquella que se compone como un montaje o ensamblaje de los tres modos señalados, llegando a ser un saber - instintivo - que, domeñando la unilateralidad de cada cual, logra conducir la historia, como un arte de la ficción o del montaje, entre el olvido y la memoria. Pues el olvido y la memoria han de ser siempre "creativos", si se quieren con sentido para la vida. En otras palabras, es esencial reconocer que la historia útil para la vida recrea el pasado, llegando a emparentarse en alguna medida con el

4 Presupone esta comprensión histórica una ontología de la potencia, en la que lo existente no es concebido como un "ente" sustancial, una cosa, sino como conatus, como potencia de ser y existir, "El conato con el que cada cosa se esfuerza en perseverar en su ser, no es nada más que la esencia actual de la misma", y su perfección coincide con su esfuerzo de ser y existir (SPINOZA, 2009, p. 133). 
arte: "el auténtico historiador debe poseer la fuerza de volver a formular lo ya conocido como algo nunca antes visto" (2013, p. 94).

Nietzsche declara en la II Intempestiva que padecemos de una cierta indigestión, causada por un "exceso de historia", traído hasta el presente por nuestra ciencia. En palabras del filósofo: "el exceso de historia ha debilitado la fuerza plástica de la vida, porque ha de comprender el servicio del pasado como un alimento vigorizante" (2013, p. 135). Así, reconoce Nietzsche, el antídoto para tal enfermedad es lo "ahistórico" y lo "suprahistórico". El primero es definido como una especie de instinto para el olvido; y el segundo como el poder de dirigir la mirada desde lo que deviene - y es considerado histórico - a lo eterno: arte y religión (2013, p. 135-136). Frente a ambos poderes, la ciencia, llevada por un camino errado, se levanta, declarando paralelamente una incapacidad natural de habérselas con el olvido y una ineptitud para medirse con lo eterno (2013, p. 136). Falta, en nuestra época ese instinto para alimentarnos de forma adecuada del pasado; carecemos de un oído musical, que permitiría interpretar - como si se tratase de una partitura - el poderío original del pasado con inaudita novedad. Porque hacer historia debiera consistir en desplegar un impulso creativo, a fin de: "parafrasear con ingenio un tema conocido, incluso habitual, una melodía cotidiana, en elevarlo y exaltarlo como símbolo universal y así dejar entrever en el tema original todo un mundo de profundo sentido, poder y belleza" (2013, p. 92). Pero para ello el hombre debe tener la fuerza de romper y de liberarse de su pasado, en otras palabras, de romper consigo mismo, fuerza destructiva que no puede sino acompañarse de un impulso creativo que conduciría al hombre a crear, a darse a sí mismo un nuevo tiempo y, por esa vía, un nuevo porvenir. La potencia de un saber al servicio de la vida se cifra, entonces, en el uso de una fuerza plástica que permite recolocar, recomponer $\mathrm{u}$ ofrecer un nuevo montaje para el pasado en la que se encuentra lo histórico y lo no-histórico. Tal es el modo de combatir la aludida indigestión. El camino, a la sazón, es educar a los hombres para la historia como se educa para la música, a fin de extender esa fuerza plástica a todos los individuos, fuerza que les permitirá equilibrarse entre el olvido y el recuerdo y, en suma, manejarse con el pasado que viene a ser lo extraño en nosotros mismos. 
Al volver, vale decir, al repetir a Nietzsche desde Deleuze, tomando en cuenta la distinción establecida entre los usos del pasado o formas de hacer historia, se podría barruntar que falta un equilibrio en el hacer filosófico para que éste se descubra útil para la vida. Inspirados, pues, por una perspectiva deleuziana, cabría pensar que la metafísica, en el campo de la filosofía, pareciera proceder de modo semejante a la historia monumental, cuando para reconocer un pensamiento como legítimo se impone como condición examinar los conceptos creados bajo la óptica de las autoridades que fundan la razón y la filosofía occidental. En este caso, la metafísica contribuye, sin lugar a dudas, a perpetuar un precioso legado, como es el de la tradición del lógos, institucionalizada por Platón y Aristóteles, que definen y cierran la discusión sobre qué discute y cómo procede la filosofía. Pero, al mismo tiempo, esta actitud "monumental" ante el pasado filosófico impide pensar o aventurarse más allá de lo pensado, reduciendo el pensamiento a un ejercicio de representación de la relación del pensamiento consigo mismo. Por otro lado, si continuamos en la misma repetición, nos encontramos con que la historia de la filosofía se comporta de manera semejante a la historia anticuaria. En su apuesta por conservar el pasado en toda su grandeza, el anticuario-filósofo no logra crear o al menos proponer una historia filosófica útil para la vida, al contrario da a luz un saber estéril, que se contenta con conservar las ideas del pasado en toda su lejanía para impedir que su grandeza se corrompa en un presente que sólo produce miserias. Finalmente, ¿qué decir de la historia crítica nietzscheana? ¿En qué actitud filosófica encontramos la fuerza de romper con el pasado? Podría proponerse, sin arriesgar demasiado, que la "teoría crítica", en especial con Adorno y Horkheimer (1998)5, corresponde a dicha tentativa. La teoría crítica es reconocida por el esfuerzo conceptual que ha puesto el acento en desnudar la miseria presente, rastreando las aberraciones del pasado - entiéndase las aberraciones

5 Dialéctica de la llustración es crucial para entender esta potencia destructiva, ejecutada expresamente contra las pretensiones de saber puro de la ciencia moderna: "El pensamiento se reifica en un proceso automático que se desarrolla por cuenta propia, compitiendo con la máquina que él mismo produce para que finalmente lo pueda sustituir. [...] El modo de procedimiento matemático se convirtió, por así decirlo, en ritual del pensamiento. Pese a la autolimitación axiomática, dicho procedimiento se instaura como necesario y objetivo: transforma el pensamiento en cosa, en instrumento, como él mismo lo denomina" (1998, p. 78-79). 
del proyecto emancipatorio de la Ilustración-, liderando en lo concreto una apuesta por derribar y desenmascarar la supuesta grandeza de la Historia/Razón de occidente. Sin embargo, tal apuesta, dominada por el trabajo de lo negativo, carece, como la historia crítica, del impulso creador que permitiría hacer sonar la historia de otro modo.

Pues bien, la falta de un equilibrio entre las tres actitudes filosóficas es sopesada por Deleuze, quien, siguiendo la propia exhortación de la segunda intempestiva, da muestras de enlazar en su pensamiento los trazos del metafísico, historiador y filósofo crítico para realizar el vitalismo nietzscheano, no legándonos una ontología vitalista (CANAVERA, 2015), como parece pensar Badiou (2000), sino un pensamiento que se ve desbordado por la vida: "un pensamiento que iría hasta el límite de lo que puede la vida, un pensamiento que conduciría la vida hasta el límite de lo que ella pueda. En lugar de un conocimiento que se opone a la vida, una pensamiento que afirmaría la vida" (DELEUZE, 1983, p. 115).

\section{Deleuze: ¿uno o múltiple?}

No es difícil reconocer en el pensamiento de Deleuze, plasmado en libros, cursos y entrevistas, el trabajo del erudito y del historiador, el rigor del metafísico o el entusiasmo del filósofo crítico o "destructor", como líneas de fuerza que se superponen y en cuyo despliegue se percibe que el filósofo no interviene en ningún caso en las arenas del puro saber, sino que recorre su afuera: afectado por los avatares de la época, no se deja seducir por las seguridades de la vida universitaria; atento a las convulsiones de un presente golpeado por hechos que llegarían a producir fuertes transformaciones sociales y políticas en Francia; sensible a las rupturas que fragmentan la aparente homogeneidad de la historia, $\mathrm{y}$, en último término, a los hechos que desgarran la vida en las fronteras del pensamiento, el trabajo de Deleuze da cuenta de un esfuerzo por poner el presente en conceptos - atendiendo a su peculiaridad: estar más allá y más acá del Estado - desde una posición política o "micropolítica" (2004a, p. 165-166) en el que se cruzan, según intentamos mostrar, metafísica, historia y crítica. 
¿Cómo se materializan estas líneas de fuerza? En el francés se hace patente una crítica a la Historia de Filosofía, pues no sólo elide pensar su propia época, sino que se caracteriza por someter conceptos e ideas a la aprobación de las grandes autoridades del pasado: "la historia de la filosofía siempre ha sido el agente de poder dentro de la filosofía. Siempre ha jugado un papel represor" (DELEUZE, 2004a, p. 17). De acuerdo a esta crítica, lo que se vuelve necesario hoy es construir otra historia, la historia de aquellos pensadores exiliados de las academias, que conducen el acto de pensar a los límites de la locura y que, por lo mismo, no son considerados dignos de la misma veneración que se le tributa a las grandes autoridades que conforman el Tribunal de la Razón de la Historia de la Filosofía: "hay para mí - escribe Deleuze - un vínculo secreto entre Lucrecio, Hume, Spinoza o Nietzsche, un vínculo constituido por la crítica de lo negativo, la cultura de la alegría, el odio a la interioridad, la exterioridad de las fuerzas y las relaciones, la denuncia del poder, etc. (1996a, p. 14). ${ }^{6}$ Asimismo, junto con el rechazo declarado a las autoridades filosóficas - sobre todo en un comienzo a Hegel - y un consiguiente apego a las figuras "menores" de la filosofía, se advierte en distintos pasajes el esfuerzo por construir una "metafísica de la multiplicidad". Esta particular metafísica, que encarna en tanto crítica la propuesta concreta de llevar a cabo un "sistema de lo múltiple" (MENGUE, 2008), vincula las componentes de esa otra historia - que tiene mucho de ficción y simulación-, trazando líneas de vecindad entre los conceptos e ideas creadas por aquellos exiliados de la Historia de la Filosofía, y, en último término, (re) crea el pasado filosófico, elevando a la enésima potencia el arte de crear conceptos, con sentido para la vida.

Ahora bien, como se quiere mostrar, en el caso de Deleuze el "historiador" y el "filósofo" no constituyen dos personajes distintos. La crítica que pone en juego este sistema de lo múltiple y esta otra historia de la filosofía, debe ser comprendida en relación directa con la creación de conceptos y la filosofía del acontecimiento: a la inversa de la metafísica

6 Esta preocupación deleuziana se entronca con la historiografía crítica inglesa, representada por Margaret Jacob (1981) y Jonathan Israel (2001), quienes elaboran la categoría de "Ilustración radical", desde la que hacen una re-lectura crítica de la historiografía de la modernidad. Una actualización de esta discusión epistemológica y política la lleva a cabo Sonja Lavaert (2014). 
tradicional, preocupada por lo que permanece y perdura, la ontología deleuziana se propone pensar y crear conceptos de aquello que hace acontecimiento y, finalmente, de lo que está en devenir, creación que concierne, en último término, con el diagnóstico de nuevas - y más adecuadas - formas de vida colectiva. Tal diferencia, pensar lo que perdura y pensar lo que deviene, pone de manifiesto la diferencia entre dos tipos enfrentados de concebir y hacer filosofía, tipos que hasta ahora han sido vinculados de manera jerárquica: por un lado, la filosofía de la representación sedentaria, que recorre y domina el panorama filosófico desde la perspectiva académica, que se arroga el poder de definir qué problema y qué pensador es digno de engrosar los anales de su Historia; y, por otro lado, un pensamiento nómade, expresivo y productivo, conducido por sujetos marginados en su mayoría de la academia, que poco parecieran tener en común, salvo un cierto tono que rompe de plano con esa tradición que se opone a pensar el cambio, el devenir y lo contingente (DELEUZE, 2004b, p. 382-383). Para Deleuze, sin embargo, ambas formas de concebir y hacer filosofía no se oponen meramente, sino que se dan como dos velocidades, dos tiempos que se solapan y que surcan el territorio de la inmanencia: la inmanencia será, en otras palabras, el lugar sin espesor de ese encuentro y conflicto entre un découpage vertical, sedentario, que organiza y jerarquiza la historia, y otro horizontal, nómade, que insiste en resaltar la heterogeneidad de esta otra historia singular. Los cruces, oposiciones y hasta tensiones que puedan darse entre ambas formas son, propiamente, los movimientos que han de ser trazados por el filósofo-historiador que deviene cartógrafo (2004b, p. 370).

La crítica que emprende el sistema de lo múltiple asimismo debe entenderse en relación directa con la creación de conceptos y el vínculo esencial que esta acción experimenta con el acontecimiento, acción que destaca a su vez el arte de "crear" y no meramente "conservar" la vida, si lo observamos ahora de la perspectiva de Nietzsche, para quien decíamos deben conjugarse las bases del conocimiento y la vida. Pero la vida que toca a la filosofía - la vida impersonal de la filosofía - es la vida de los conceptos o la vida del acontecimiento que es capaz de captar y, luego, de crear conceptualmente: "El concepto filosófico no se refiere a lo vivido, por compensación, sino que consiste, por su propia creación, en 
establecer un acontecimiento que sobrevuela toda vivencia tanto como cualquier estado de las cosas. Cada concepto talla el acontecimiento, lo perfila a su manera [...]" (DELEUZE, 1993, p. 22). Por ello, en tanto creación, el concepto puede y debe ser comprendido como una forma de conocimiento: "El concepto es evidentemente conocimiento [...] y lo que conoce, es el acontecimiento puro, que no se confunde con el estado de cosas en el que se encarna." (DELEUZE, 1993, p. 37).

La crítica que lleva adelante el sistema de lo múltiple y esa otra historia muestra que cuando la filosofía depone su actividad creativa y se convierte en instrumento de un poder (que organiza la historia como un cuerpo de ideas y conceptos, apelando a una trascendencia - razón, dios, etc.) sirve, entonces, a la mera conservación de la vida y su tiempo ad-hoc, el presente. Contra esta deposición - y a la larga depotenciación de la filosofía - Deleuze afirma y rastrea los meandros de esa otra historia, nunca acabada, que reconoce en Spinoza y Nietzsche los hitos más cercanos a su obra (DELEUZE, 2004a, p. 20; ANTONELLI, 2013), y cuyas principales características son: hacer hablar un yo impersonal, que se abre paso para ser la expresión del acontecimiento que desgarra la unidad de obra y autor, renunciando, en sus palabras, a toda imagen dogmática del pensamiento (DELEUZE, 2002, p. 204), para experimentar allende la Historia, que nos separa de nosotros mismos, lo intempestivo (DELEUZE, 1996a, p. 154-155).

\section{Diagrama: experimentar entre las fuerzas y vivir para contarlo}

Si Nietzsche declara la importancia de un oído musical para la historia, Deleuze a su turno invoca para la filosofía la utilidad de otra disciplina artística, la pintura, específicamente el collage, entendido como técnica de ensamblaje de elementos heterogéneos e instrumento que permite crear una cosa nueva (2002, p. 18). Lo que está puesto en juego no es una mera analogía. La pintura enseña a la filosofía la necesidad de la catástrofe (DELEUZE, 2007c, p. 26). Pero la pintura no representa un modelo para la filosofía, tampoco el cine o la música. Lejos 
de esto, arte y filosofía tienen un punto común, que es el de conectar el pensamiento con su afuera. Y para ello lo primero, sea en la filosofía o en la pintura, es cortar los lazos con la representación ordenada y orgánica del mundo, abrirse al caos, pasar por la catástrofe, dejarse coger por las fuerzas del afuera y apostar por ese "germen de orden" que luego comienza a nacer en la hoja en blanco, en la partitura o en la tela (DELEUZE, 2001, p. 120). Para Deleuze, observando la pintura, el diagrama encarna, pues, un tipo de "caos-germen": un caos del que algo sale (DELEUZE, 2007c, p. 90).

La relación de la filosofía con su afuera se produce, al igual que se observa en el arte, como un cuestión "clínica", es decir, como el intento por liberar la vida de aquello que la aprisiona: el poder, el organismo, el hombre, etc. Deleuze se pregunta en Crítica y clínica (1993): “¿qué salud bastaría para liberar la vida allá donde esté encarcelada por y en el hombre, por y en los organismos?" (1996b, p. 16). Se invoca aquí "salud" como un término que es clave en la disputa con el organismo, específicamente, con la forma-organismo, que corta o reduce el vínculo de seres e individuos con su afuera (LANDAETA; ESPINOZA, 2014). Saludable sería, en tales términos, asumiendo un riesgo consustancial, abrirnos al influjo de las fuerzas del afuera, como el arte de manera diferenciada se abre al afuera en la pintura o la literatura. Un diagrama filosófico, entonces, constituiría ese breve equilibrio que nos protege en el instante de estar en medio de las fuerzas para crear algo nuevo.

En el tránsito hacia el afuera, la filosofía muestra que no sólo posee un vínculo preponderante con la no-filosofía, sino con las distintas disciplinas que buscan liberar la vida donde sea vea oprimida o bloqueada. Y, si volvemos a Nietzsche, este tránsito hacia el afuera es también decisivo, porque es testimonio de que se posa en el pensamiento filosófico una falta constitutiva: la filosofía no puede girar sobre sí misma sin enfermar de un exceso, comparable al que causa la "indigestión histórica" detectada en la II Intempestiva. Por ello, la filosofía solo puede ser invaginación del afuera que fuerza a pensar, mostrando, a su vez, que el afuera es un pliegue del pensamiento y que el pensamiento sólo puede darse, como destaca Manola Antonioli, entre el sujeto y el mundo (1999, p. 34). Porque pensar no es una actividad "interior", 
así como el afuera es siempre un pliegue del pensamiento y no una presencia "exterior" (MONTEBELLO, 2008, p. 51-52). El pensamiento se produce como una afección de las fuerzas del afuera, ya que son las fuerzas del afuera las que ponen el pensamiento en estado de exterioridad (ZOURABICHVILI, 2004, p 46-47). Estar "en medio" (DELEUZE, 2004b, p. 29), consiste, a la sazón, en estar "entre las fuerzas". Y entre las fuerzas se halla pues el inconsciente, campo de fuerzas informal, allende toda forma y representación (DELEUZE, 2005, p. 141). Indagar acerca de la relación de las fuerzas y el pensamiento nos lleva a bosquejar algunas ideas sobre el diagrama en filosofía.

Presente transversalmente en su obra, Deleuze propone que el diagrama no es un método, sino más bien una práctica, que busca producir el encuentro del pensamiento y el afuera. Si el problema común al arte y la filosofía consiste en hacer visibles fuerzas que no lo son, todo comienza con el trazado de un diagrama tentativo: siendo las líneas los elementos constitutivos de las cosas y de los acontecimientos, cada cosa tendrá su propia cartografía o diagrama (DELEUZE, 1996b, p. 56). En Foucault, Deleuze declara que el diagrama no sirve para representar un mundo, sino que al contrario organiza o "produce un nuevo tipo de realidad" (1987, p. 62). Un mundo objetivado sería el objeto de la filosofía "representativa", ya que mientras el método engendra un pensamiento que busca ser fiel a la interioridad de la conciencia, el diagrama se presenta como una experiencia rigurosa, pero tocada por lo inconsciente: el diagrama a fin de cuenta resulta ser: "el elemento informe de fuerzas" (DELEUZE, 1987, p 70). Llegamos, pues, con este último texto citado, a un punto problemático, cargado de ambigüedad: el diagrama designa, por un lado, este "mínimo de orden" que permite a la filosofía, en tanto saber útil para la vida, protegerse del caos en su tránsito al afuera; y, por otro lado, el propio diagrama designa la fórmula estratégica del poder o, incluso, el elemento o lugar, más bien, el no-lugar del poder (DELEUZE, 2008, p. 231), como apunta Philippe Mengue: “El poder es una acción sobre acciones. Pero estas relaciones suponen un elemento informal como espacio de su encuentro, el diagrama. Las fuerzas se encuentran a partir de y en él; se conjugan, según tal o cual relación, tramando uniones en perpetuo devenir" (2008, p. 405). La ambigüedad, 
sin embargo, es constitutiva, como lo afirma el propio Deleuze (2007a, p. 118) y como bien lo destaca Éric Alliez (2013). Pues, si el problema común al arte y la filosofía es el problema de las fuerzas; si el arte busca "captar las fuerzas"; si la filosofía se produce como una "afección de las fuerzas del afuera"; y si para el arte y la filosofía lo esencial mediante el diagrama es: "hacer visible las fuerzas del afuera", ofreciendo contra la representación una práctica de resistencia, de contrapoder, es porque el propio poder en su componente creativo es diagramático: “[...] la filosofía puede asumir el papel de contrapoder, a condición a condición de que la filosofía deje de pensarse como profecía [...] y se dé como tarea analizar, elucidar, hacer visible y, por lo tanto, intensificar las luchas que se desarrollan en torno al poder" (FOUCAULT, 1999, p. 117).

“El diagrama es el poder" (2014, p. 78), afirma Deleuze en el curso sobre Foucault el año 1986. Por tanto, si, como piensa Foucault, el poder es "microfísico" (DELEUZE, 2008, p. 228-229), el diagrama o los diagramas, serán su elemento. Cabe establecer, entonces, el encuentro de dos diagramas que se enfrentan (DELEUZE, 2007a, p. 128): mientras que el diagrama del lado del poder es cualquier multiplicidad a controlar (DELEUZE, 1987, p. 101), el diagrama, del lado de la filosofía y el arte, constituye ese mínimo de orden que requiere todo acto creativo y, en ese sentido, puede ser definido por una experiencia, la experiencia de abismarse al afuera, protegiéndose del caos, para captar las fuerzas "en estado puro" y llegar así a entrever nuevas posibilidades de vida (DELEUZE, 1993). El francés es enfático en afirmar que: "la vida deviene resistencia al poder cuando el poder tiene por objeto la vida" (1987, p. 122). Y para comprender de modo más claro esta afirmación, se puede echar mano a una última consideración sobre las fuerzas hecha por Deleuze, nuevamente tomando en cuenta una apreciación sobre la filosofía de Foucault. En el artículo inédito del año 1984: Sobre los principales conceptos de Foucault, que deviene más tarde la base del texto Foucault, Deleuze afirma que el diagrama, en primer lugar, en tanto determinación de un conjunto de relaciones de fuerza, no agota la fuerza, ya que ésta puede entrar siempre en otras relaciones y composiciones; en segundo lugar, que si bien el diagrama proviene del afuera, el afuera no se confunde con un diagrama particular, ya que éste no cesa de "trazar" 
nuevos diagramas. En la teoría del poder la resistencia es primera, hallándose en directa relación con el afuera (DELEUZE, 2007b, p. 229-231). En efecto, para el francés la fuerza tiene un potencial en relación con el diagrama en el que está momentáneamente "capturada", y ese potencial - para cuando Deleuze repite a Foucault - viene a ser la resistencia que hace posible la existencia del cambio y, en último término, la emergencia de una nueva atmósfera para la vida.

\section{Consideraciones finales}

Para componer un vitalismo deleuziano no bastaba con analizar el vitalismo como un concepto más. Por este motivo, se propuso leer el vitalismo deleuziano desde la mutua implicancia que tienen en su pensamiento las figuras del "historiador", el "metafísico" y el "crítico". La unión de tales figuras era derivada de la repetición de la II Intempestiva de Nietzsche, que nos advertía sobre la importancia de encontrar un equilibrio entre memoria y olvido para luchar contra el presente en espera de un nuevo porvenir para vida. Para Deleuze, como para Nietzsche, lo esencial concretamente consiste en producir una nueva salud, luchando contra las miserias del presente. Y si Nietzsche invoca un "sentido musical", que permitiría cultivar el saber histórico, Deleuze invoca a su turno el diagrama, de gran importancia para la pintura (2001), que testimonia el tránsito del acto pictórico por la catástrofe, cuando se trata de hacer visible las fuerzas invisibles del caos. Un diagrama para la filosofía, constituiría un medio para hacer visibles las luchas alrededor del poder que buscan, precisamente, liberar la vida. Pero el drama de esta lucha, que es también el drama del presente, es que las propias relaciones de poder tienen la forma del diagrama y, por ello, eminentemente, algo de su plasticidad. Y si la función clínica de filosofía y arte pasa por diagnosticar nuevas posibilidades de vida, ésta, la clínica, no puede pensarse al margen, sino envuelta en unas relaciones de poder, que tanto signan su captura como su liberación ¿Cómo procurar nuevas posibilidades de vida? Esta pregunta intentan responder el artista y el filósofo, cada uno a su manera: "inventar formas de vida capaces de resistirse al poder y hurtarse al saber, aunque el saber intente penetrarlas y el poder 
intente apropiárselas" (DELEUZE, 1996a, p. 151), conscientes, sin embargo, de la amenaza que ejercen por un lado los diagramas de poder $y$, por otro lado, la muerte y la locura que llama a la puerta en la experimentación con las fuerzas del afuera.

\section{Referencias}

DELEUZE, G. Nietzsche et la philosophie. París: PUF, 1983.

DELEUZE, G. Diferencia y repetición. Buenos Aires: Amorrortu, 2002.

DELEUZE, G. Lógica del sentido. Buenos Aires: Paidós, 2005.

DELEUZE, G. Écrivain non: Un nouveau cartographe. Critique, t. 31, n. 343, p. 1207-1227, 1975.

DELEUZE, G. Deseo y Placer. En, Gilles Deleuze. Dos Regímenes de Locos. Textos y entrevistas (1975-1995). Valencia: Pre-Textos, p. 223-236, 2007a.

DELEUZE, G.; PARNET, C. Diálogos. Valencia: Pre-Textos, 2004a.

DELEUZE, G.; GUATTARI, F. Mil Mesetas. Capitalismo y esquizofrenia 2. Valencia: Pre-Textos, 2004b.

DELEUZE, G. Francis Bacon: Lógica de la sensación. Madrid: Arena Libros, 2001. DELEUZE, G. El poder: curso sobre Foucault. Tomo II. Buenos Aires: Cactus, 2014. DELEUZE, G. Sobre los principales conceptos de Michel Foucault. En, Gilles Deleuze. Dos Regímenes de Locos. Textos y entrevistas (1975-1995). Valencia: PreTextos, p. 223-236, 2007b.

DELEUZE, G. Foucault. París: PUF, 1987.

DELEUZE, G. Conversaciones (1972-1990). Valencia: Pre-Textos, 1996a.

DELEUZE, G.; GUATTARI, F. ¿Qué es la filosofía? Barcelona: Anagrama, 1993.

DELEUZE, G. L'immanence: une vie. Philosophie, n. 47, p. 3-7, 1995.

DELEUZE, G., Pintura. El concepto de diagrama. Buenos Aires: Cactus, 2007c. 
ADORNO, T.; HORKHEIMER, M. Dialéctica de la Ilustración. Fragmentos filosóficos. Madrid: Trotta, 1998.

AGAMBEN, G. Homo Sacer I, El poder soberano y la nuda vida. Valencia: Pre-textos, 1998.

ALLIEZ, É. Ontology of the Diagram and Biopolitics of Philosophy. A Research Programme on Transdisciplinarity. Deleuze studies, v. 7, n. 2, p. 217-230, 2013.

ANTONELLI, M. Vitalismo y desubjetivación. La ética de la prudencia en Gilles Deleuze. Signos Filosóficos, v. XV, n. 30, julio-diciembre, p. 89-117, 2013.

ANTONIOLI, M. Deleuze et l'Histoire de la philosophie. París: Kimé, 1999.

BADIOU, A. Of Life as a Name of Being, or, Deleuze's Vitalist Ontology'. Pli, n. 10, p. 191-199. 2000.

CANAVERA, J. El vitalismo de Gilles Deleuze. Daimon. Revista Internacional de Filosofía, n. 66, p. 135-150. 2015.

FOUCAULT, M. Vigilar y castigar. Nacimiento de la prisión. Buenos Aires: Paidós, 2002.

FOUCAUlT, M. Anti-Oedipus. Capitalism and Schizophrenia. Minneapolis: University of Minnesota PRESS, 2000.

FOUCAULT, M. La filosofía analítica de la política. En, Estética, ética y hermenéutica. Obras esenciales, v. 3, p. 111-128. 1999.

FOUCAULT, M. El nacimiento de la biopolítica, Curso en el Collège de France (19781979). Buenos Aires: FCE, 2007.

GROS, F. Le Foucault de Deleuze: une fiction métaphysique. Philosophie, n. 47, p. 53-63, 1995.

GUATTARI, F; ROLNIK, S. Micropolítica. Cartografía del Deseo. Madrid: Traficante de sueños, 2006.

ISRAEL, J. Radical Enlightenment. Philosophy and the Making of Modernity 1650-1750. Oxford: Oxford University Press, 2001.

ESPOSITO, R. Bíos: biopolítica y filosofía. Buenos Aires: Amorrortu, 2006.

HARDT, M.; NEGRI, A. Imperio. Barcelona: Paidós, 2005. 
JACOB, M. The Radical Enlightenment: Pantheist, Freemasons and Republicans. Louisiana: Cornerstone,1981.

LANDAETA, P.; ESPINOZA, R. Geofilosofía de la ciudad para pensar más allá del organismo. Revista de Filosofía Aurora, Curitiba, v. 26, n. 38, p. 295-313, 2014.

LAVAERT, S. Radical enlightenment, enlightened, subversion and Spinoza. Philosophica, n. 89, p. 49-102. 2014.

MENGUE, P. Deleuze o el sistema de lo múltiple. Buenos Aires: Las cuarenta, 2008. MONTEBELLO, P. Deleuze. La passion de la pensée. París: Vrin, 2008.

NIETZSCHE, F. Sobre la Utilidad y el perjuicio de la historia para la vida [II Intempestiva]. Madrid: Biblioteca Nueva, 2013.

PROTEVI, J. Deleuze and life. En, Smith, D.; Somers-Hall, H. (comp.). Cambridge Companion to Deleuze. Cambridge: Cambridge University Press, 2012.

SPINOZA, Ética demostrada según el método geométrico. Madrid: Trotta, 2009.

SOTO GARCÍA, P. Conflicto y vida: La recuperación del Centauro en el pensamiento de Roberto Esposito. En, Revista de la Academia, v. 20, Primavera, p. 67-48. 2015.

SHERER, R. Miradas sobre Deleuze. Buenos Aires: Cactus, 2012.

ZOURABICHVILI, F. Deleuze. Une philosophie de l'évènement. En, Zourabichvili, F.; Sauvagnargues, A.; Marrati P. La philosophie de Deleuze. París: PUF, 2004.

ZOURABICHVILI, F. Six notes sur le percept. Sur le rapport de la critique et de la clinique chez Deleuze. En, Gilles Deleuze, la logique du sensible. Lille: De l'Incidence, p. 375-404, 2013.

Recibido: 03/11/2016

Recebido: 03/11/2016

Aprobado: 22/01/2017

Aprovado: 22/01/2017 